\title{
Demonstration of Cephaloceles in Patients with Rhinorrhea: Single Center Experience of Intrathecal Gadolinium Enhanced MR Cisternography
}

\author{
Hakan Cebeci $\mathbb{B}^{1,{ }^{*}}$, Cem Bilgin ${ }^{2}$, Selman Candan $^{2}$, Selcuk Yilmazlar ${ }^{3}$ and Bahattin Hakyemez ${ }^{2}$ \\ ${ }^{1}$ Department of Radiology, Selcuk University Medical Faculty, Konya, Turkey \\ ${ }^{2}$ Department of Radiology, Bursa Uludag University Medical Faculty, Bursa, Turkey \\ ${ }^{3}$ Department of Neurosurgery, Bursa Uludag University Medical Faculty, Bursa, Turkey \\ "Corresponding author: Department of Radiology, Selcuk University Medical Faculty, 42130, Konya, Turkey. Tel: +90-3322415000, Email: dr.hakancebeci@gmail.com
}

Received 2019 March 25; Revised 2019 November 27; Accepted 2020 February 05.

\begin{abstract}
Background: Magnetic resonance (MR) cisternography has the crucial role for diagnosis of cerebrospinal fluid (CSF) leakage in patients with rhinorrhea and otorrhea. Trauma is the major cause of rhinorrhea. Cephalocele is diagnosed in some rhinorrhea patients.

Objectives: To evaluate the CSF leakage in patients with rhinorrhea and assess the frequency of cephalocele in the etiology of rhinorrhea.

Patients and Methods: Intrathecal gadolinium enhanced MR cisternography and nonenhanced CT of paranasal sinus images of patients with suspected CSF rhinorrhea between October 2012 and September 2018 were evaluated retrospectively. Twenty-one patients with the diagnosis of contrast leakage causing rhinorrhea were included in the study. All patients had intrathecal gadolinium enhanced MR cisternography. Three dimensional (3D)-T1 weighted and 3D-fluid attenuated inversion recovery (FLAIR) head MRI was obtained after administration of $1 \mathrm{ml}$ intrathecal gadoterate meglumine after half, 3 , and 8 hours. Locations of contrast leakage and etiologies were analyzed.

Results: Patient group consisted of 21 patients with persistent or intermittent rhinorrhea. CT imaging showed bone defect in all patients. Intrathecal gadolinium enhanced MR cisternography revealed CSF leakage (10 ethmoid, 10 sphenoid, and 1 frontal). Cephalocele was detected in 10 of 21 patients.

Conclusion: MR cisternography with intrathecal gadolinium enhancement is an effective and safe imaging modality compared to other techniques used for diagnosis of CSF leakage. Accurate localization of CSF fistula and demonstration of herniating content from cranial bone defect is feasible with this technique.
\end{abstract}

Keywords: Cerebrospinal Fluid, Magnetic Resonance Imaging, Cisternography, Cephalocele

\section{Background}

The term 'cephalocele' refers to extracranial extension of meninges with or without brain tissue. Cephaloceles consist of two types. It is called meningocele when only meninges are involved, and it is called meningoencephalocele if the cerebral tissue protrudes with meninges. Cephaloceles in the anterior cranial base may lead to spontaneous cerebrospinal fluid (CSF) leakage to the sinonasal cavity, called rhinorrhea. CSF leaks are mostly traumatic (accidental or iatrogenic). Only 3\% - 4\% of CSF leaks are spontaneous and female/ male ratio is $2 / 1$ (1).

Accurate localization of CSF fistulas has an important role in the surgical treatment approach. Presurgical localization increases the chance for proper dural repair and could prevent complications (2). Successive surgical repair of dural tear is also needed to prevent recurrent meningitis (3).

Several imaging techniques are used for detection of proper leakage location. Precise localization of CSF leakage still remains a challenge despite advances in imaging for neuroradiologists. Computed tomography (CT) of paranasal sinuses, intrathecal contrast enhanced CT (CT-cisternography) and heavily T2 weighted magnetic resonance (MR) imaging and intrathecal gadolinium enhanced MR cisternography (Gd-MR cisternography) are mostly used modalities in clinical routine (4). CT has superiority to detect bone defects in the cranium base, but it cannot directly reveal the CSF leakage, which is the ma- 
jor limitation of CT. CT gives false positive results with bone defects without meningeal tear (5). CT cisternography can also show the leak localization, but ionizing radiation and the application of iodine-based contrast material are the major disadvantages of this technique. Intrathecal injected iodinated contrast material cannot disperse in CSF freely causing false negative results. In addition, it has adverse effects including arachnoiditis, epilepsy, and allergic reactions $(6,7)$.

Gd-MR cisternography is a well-known minimal invasive imaging technique in suspected rhinorrhea. Several reports in the literature described its higher diagnostic sensitivity and specificity in localizing CSF leakage $(5,7,8)$.

\section{Objectives}

This study aimed to present our experience of Gd-MR cisternography in patients with rhinorrhea, and particularly evaluate the frequency of cephaloceles among rhinorrhea patients.

\section{Patients and Methods}

All Gd-MR cisternography procedures performed between October 2012 and September 2018 were evaluated retrospectively. This study was approved by the local ethics committee. Indications for MR cisternography in our department were rhinorrhea, otorrhea and idiopathic intracranial hypotension, and arachnoid cysts. Patients suffering from persistent or intermittent rhinorrhea and with positive Gd-MR cisternography findings were included in the study. Gadolinium leakage from subarachnoid spaces to paranasal sinuses was accepted as the positive finding for rhinorrhea. Twenty five patients underwent Gd-MR cisternography in the study period, and four patients with suspected rhinorrhea had normal Gd-MR cisternography. A total of 21 patients in whom extravasation from subarachnoid space to paranasal sinuses or nasal cavity was detected, were included. Sixteen of 21 patients also had non-contrast CT examination of paranasal sinuses. Localization of extravasation and causes of CSF leakage were analyzed. Beta2-transferrin test was investigated via database records of our institute.

Patients suffering from suspected rhinorrhea lasting more than 1 month were referred from otorhinolaryngology and/or neurosurgery departments. In these patients, Gd-MR cisternography was carried out in our neuroradiology department after informed consent. Claustrophobia and uncorrectable coagulopathy were accepted as contraindications for the procedure. Before acquisition of MR images, $1 \mathrm{~mL}$ gadoterate meglumine (dotarem) was injected intrathecally with a $22 \mathrm{G}$ Chiba needle under sterile conditions at L4 - L5 level. Intrathecal injections were executed with fluoroscopy guidance while patients were in lateral decubitus position by bringing the head and legs closer to the abdomen. Local anesthesia was not administered. After the injection of intrathecal gadolinium, patients were transferred to the MRI scanner in prone and $30-40^{\circ}$ head down position to increase the transport of contrast material to basal cisterns and sulci in the frontal basal region. All patients were observed for 24 hours after intrathecal injection to assess for adverse effects. MRI protocols included sagittal 3D isotropic T1-weighted turbo field echo sequence (3D isotropic T1W-TFE) with the following imaging parameters: repetition time (TR)/echo time (TE), 8.05 to 8.25 milliseconds/3.65 to 3.75 milliseconds; flip angle, $9^{\circ}$; matrix, $240 \times 240$; field of view, $220 \times 236 \times$ 179; sensitivity encoding factor, 1 ; slice thickness, $1 \mathrm{~mm}$, and sagittal 3D-Flair with the following imaging parameters TR/TE, 4800/275 milliseconds; flip angle, 90 ; matrix, $224 \times 224$; sensitivity encoding factor, 1 ; inversion time, 1650 milliseconds; slice thickness, $1 \mathrm{~mm}$. Images were obtained with a clinical 3 Tesla MR imaging system (PhilipsAchieva3T, Best, Netherlands) using 32 channel sensitivity encoding head coil.

Obtained Gd-MR cisternography and also nonenhanced paranasal CT images (scanned before MR imaging) were transferred to a workstation (GE Advantage Workstation; GE Healthcare, Milwaukee, WI) and evaluated by two neuroradiologists with 15 and 5 years of experience with consensus agreement. Leakage of gadolinium-based contrast material on MR imaging and bone defects on CT imaging were analyzed. All statistical analyses were performed with commercially available software (SPSS 16.0 for Windows; SPSS Inc, Chicago, IL). The data were entered into Excel spreadsheets (Microsoft, Redmond, WA).

\section{Results}

The patient group consisted of 21 patients (15 female and 6 male) between 16 and 59 years of age (mean age: 43.5). Demographic data and CSF leak sites are demonstrated in Table 1. Beta2-transferrin test was positive in 15 patients, negative in two patients and was not performed in four patients. In all 21 patients, gadoterate meglumine entered basal cisterns and sulci in the frontal basal region after intrathecal injection successfully. No complication related to intrathecal gadolinium injection was detected during the first 24 hours after the procedure or during the follow-up period. CSF leakage from subarachnoid space was observed objectively by two neuroradiologists (15 and 5 years experienced) with consensus. Location of leakage was frontal sinus in one patient, sphenoid sinus in 10 , and ethmoidal sinus in 10 patients. In the evaluation of CT images, there were bone defects in 12 of 16 pa- 
tients and the localization of bone defects were consistent with the CSF leakage sites in Gd-MR cisternography. Two patients with bone defects in the frontal region did not show positive Gd-MR cisternography finding for CSF leakage. Ten (47.6\%) of the patients had a cranial trauma, five $(23.8 \%)$ had an endoscopic sinus surgery history, one (4.8\%) had a history of rhinorrhea after suppurative meningitis, while five patients (23.8\%) were accepted as spontaneous rhinorrhea. Cephalocele was detected in 10 patients (47.6\%), of which seven were meningocele and three were meningoencephalocele. One of these cephaloceles was spontaneous, while five were traumatic, three were secondary to endoscopic surgery and one meningocele was developed secondary to infectious meningitis. In two traumatic meningoencephaloceles, one patient had meningoencephalocele via a defect in the lateral wall of the sphenoid sinus (Figure 1), and the other one was through ethmoidal air cells (Figure 2). In one patient with a history of recurrent meningitis, CSF leakage was detected through the cribriform plate (Figure 3). Eight of 10 cephaloceles were detected in endoscopic surgery for dural repair, while two of the cephaloceles refused operation. Sixteen of 21 patients underwent endoscopic closure of the dural tear. On the other hand, five patients refused endoscopic surgery and they are still on follow-up.

\section{Discussion}

Cephalocele (congenital or acquired), is defined as protruding of the meninges with or without cerebral parenchyma (1). Cephaloceles protruding through the sinonasal cavity can present with rhinorrhea, nasal obstruction, and recurrent meningitis. Therefore, surgical repair of the dura is mandatory for treatment. Radiological detection of the dural tear and the location of CSF leakage is important, because it increases the chance of successful surgical approach (2). Several imaging methods have been evolved for diagnosis of CSF leakage location. Frequently, un-enhanced CT of paranasal sinuses is the first radiological technique, requested by clinicians in patients with suspected rhinorrhea. CT has advantages and disadvantages in the diagnosis of CSF leakage location. It is easily accessible in many times, fast and cheap regarding to MR imaging. Bony defects in the anterior cranial fossa are best revealed with CT. But this is an indirect sign, and CT images do not show CSF leak directly. Bony defect and CSF leak location does not overlap in all patients. However, in patients with multiple bone defects, it is difficult to guess where the leakage is (9). Because of these limitations, CT imaging is commonly combined with other modalities in many institutions. We use CT imaging in combination with Gd-MR cisternography in our center $(7,8,10)$.
CT cisternography is a widely used technique worldwide. Nevertheless, CT cisternography has also disadvantages including low sensitivity in low flow fistulas, ionizing radiation exposure and adverse effects of intrathecal administration of the iodinated contrast material. Iodinated contrast materials do not spread diffusely in CSF, decreasing the sensitivity of CT cisternography. A study conducted by Goel et al. reported 38\% sensitivity of CT cisternography, while three dimensional constructive interference in steady state (3D-CISS) had $75 \%$ and intrathecal gadolinium enhanced MR cisternography had $89 \%$ sensitivity (4). Diffuse infiltration of gadolinium in CSF gives rise to high sensitivity of Gd-MR cisternography.

MR imaging without contrast enhancement is another radiologic technique used in patients with rhinorrhea. Heavily T2 weighted 3D-constructive interference in steady state (CISS) is the sequence used for unenhanced MR cisternography. However, 3D-CISS images have a high false negative and false positive results. In the study performed by Hegarty et al., there were $40 \%$ false positive results with unenhanced MR-cisternography method. Particularly, when there is an accompanying paranasal sinus inflammation, false positive results may occur. As a result of its higher rates of false positive and negative results, we do not use 3D-CISS in our routine clinical practice. Even so, its sensitivity is higher than CT cisternography $(9,11)$.

Gd-MR cisternography is a minimally invasive diagnostic method with intrathecal gadolinium injection. First studies of Gd-MR cisternography were on animal models $(12,13)$. Subsequently, various human studies reported highest sensitivity, specificity, and accuracy ratios with GdMR cisternography in the detection of CSF leakage in patients with rhinorrhea $(4,7,14,15)$. In many institutes, GdMR cisternography is applied to detect CSF leakages in patients suspected with rhinorrhea and otorrhea. However, there is no consensus on the amount and type of intrathecal gadolinium-based contrast material. According to a review report analyzing 33 articles about Gd-MR cisternography (16), Gadopentate dimeglumin (Magnevist) with the range of 0.2 - $1 \mathrm{~mL}$ was used in 29 studies, three studies used gadodiamide (Omniscan) with the range of $1-2 \mathrm{~mL}$ and gadobutrol (Gadovist) $(1 \mathrm{~mL}$ ) was used in one study. We used $1 \mathrm{~mL}$ gadoterate meglumine (Dotarem) to reveal CSF leakage. To our knowledge, in the literature, there is only one report that evaluated intrathecal injection of gadoterate meglumine. According to this report by Ozturk et al., there was no measurable signal intensity changes in dentate nucleus and globus pallidus after a single dose injection of gadoterate meglumine (17). Similar with this report, we also did not encounter any major adverse effects related to intrathecal gadoterate meglumine injection.

CSF fistulas are commonly a result of head trauma. 80\% of CSF leaks were post-traumatic, while $17 \%$ were secondary 

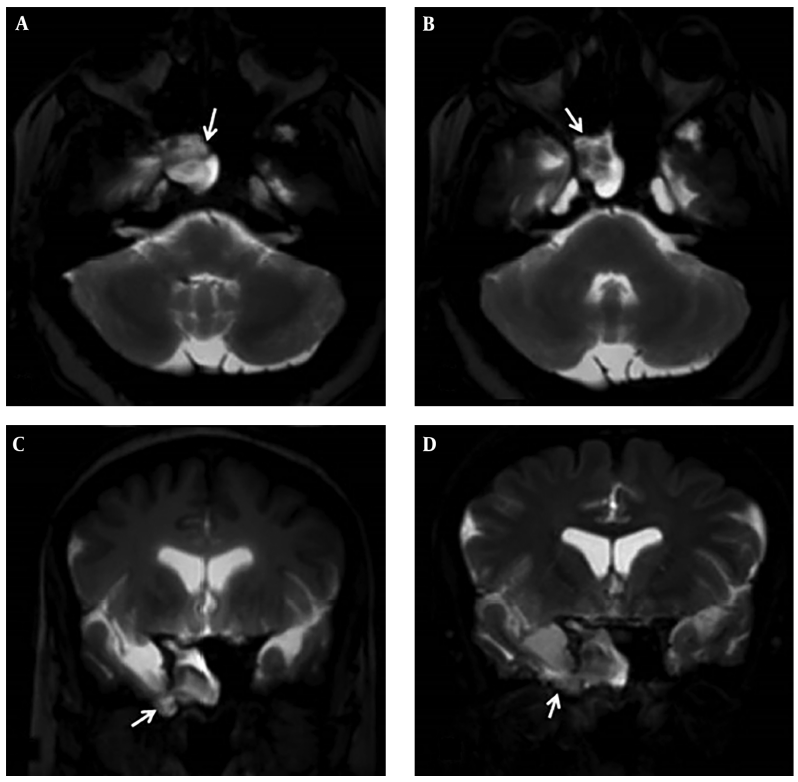

Figure 1. Intrathecal gadolinium enhanced MR cisternography examination in a 52-year-old female patient with post-traumatic persistent rhinorrhea. Axial and coronal three-dimensional T1 Weighted (3D-T1W) (A, C), and axial and coronal three dimensional fluid attenuated inversion recovery (FLAIR) (B, D) images showing hyperintense cerebrospinal fluid and accompanying temporal lobe hernia in sphenoid sinus, concordant with meningoencephalocele.
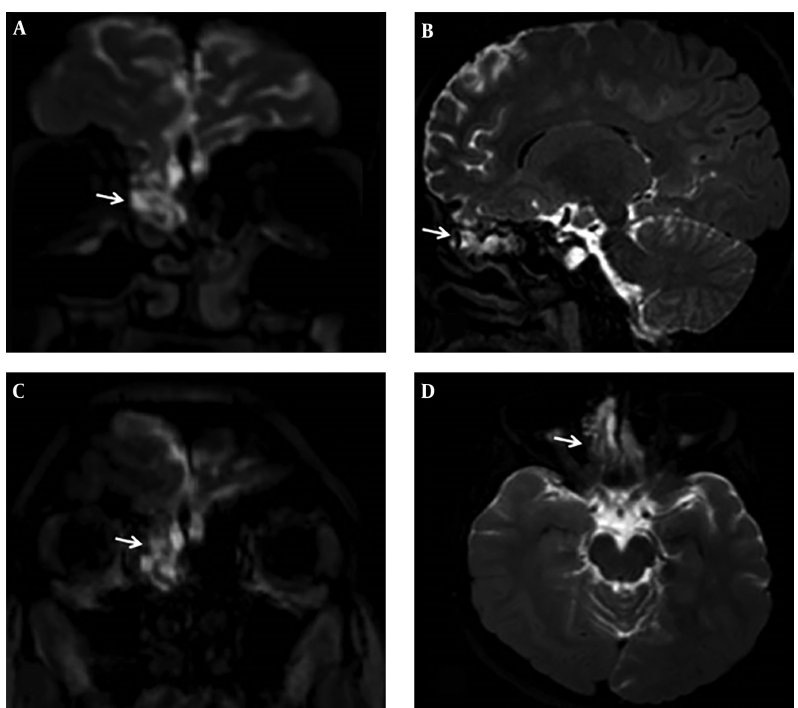

Figure 2. Intrathecal gadolinium enhanced MR cisternography examination in a 16-year-old female patient with post-traumatic persistent rhinorrhea. Coronal and sagitta three dimensional fluid attenuated inversion recovery (FLAIR) (A, B) images, and coronal and axial three dimensional T1 weighted (3D-T1W) (C, D) images showing hyperintense cerebrospinal fluid and accompanying frontal lobe hernia into ethmoid air cells.

to endoscopic surgical interventions. Only 3\% - 4\% were spontaneous (18). Etiologies of rhinorrhea in our patient group were similar to the literature. Cephaloceles of anterior cranial base commonly cause rhinorrhea when dural tear occurs. In case of a cephalocele, as a cause of rhinorrhea, patients may present with nasal obstruction. En- doscopic examination may reveal smooth polypoid mass in the nasal cavity. In this manner, radiological evaluation of rhinorrhea exhibits more importance. Because biopsy or removal of the polypoid mass in the nasal cavity is contraindicated, it may contain meninges and brain parenchyma (19). None of the patients in the study group 

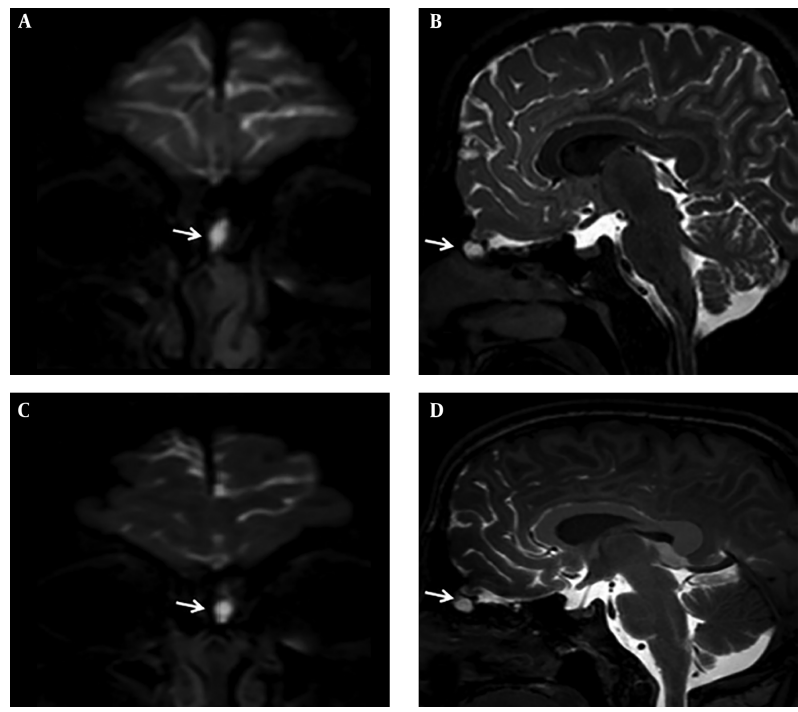

Figure 3. Intrathecal gadolinium enhanced MR cisternography examination in a 22-year-old male patient with persistent rhinorrhea. Coronal and sagittal three dimensional fluid attenuated inversion recovery (FLAIR) (A, B) images, and coronal and sagittal three dimensional T1 weighted (3D-T1W) (C, D) images showing a cystic pouch filled with gadolinium based contrast material, accepted as meningocele.

\begin{tabular}{|c|c|c|c|c|c|c|}
\hline Patient No. & Age & Gender & Etiology & Outcome & Cephalocele & Leakage site \\
\hline 1 & 52 & F & Trauma & Operated and cured & Meningoencephalocele & Lateral sphenoid \\
\hline 2 & 48 & M & Trauma & Operated and cured & - & Cribriform plate \\
\hline 3 & 16 & $\mathrm{~F}$ & Trauma & Operated and cured & Meningoencephalocele & Cribriform plate \\
\hline 4 & 22 & M & Meningitis & Operated and cured & Meningocele & Cribriform plate \\
\hline 5 & 41 & $\mathrm{~F}$ & Iatrogenic & Operated and cured & Meningocele & Cribriform plate \\
\hline 6 & 32 & $\mathrm{~F}$ & Iatrogenic & Operated and cured & - & Cribriform plate \\
\hline 7 & 43 & $\mathrm{~F}$ & Trauma & Operated and cured & - & Lateral sphenoid \\
\hline 8 & 54 & $\mathrm{~F}$ & Spontaneous & Operated and cured & - & Cribriform plate \\
\hline 9 & 46 & M & Trauma & Follow up & - & Midline sphenoid \\
\hline 10 & 53 & $\mathrm{~F}$ & Trauma & Operated and cured & Meningocele & Midline sphenoid \\
\hline 11 & 59 & $\mathrm{~F}$ & Spontaneous & Operated and cured & Meningocele & Cribriform plate \\
\hline 12 & 37 & M & Iatrogenic & Follow up & Meningocele & Cribriform plate \\
\hline 13 & 28 & $\mathrm{~F}$ & Trauma & Follow up & - & Frontal \\
\hline 14 & 56 & $\mathrm{~F}$ & Trauma & Follow up & Meningocele & Midline sphenoid \\
\hline 15 & 33 & $\mathrm{~F}$ & Iatrogenic & Operated and cured & - & Cribriform plate \\
\hline 16 & 45 & $\mathrm{~F}$ & Spontaneous & Operated and cured & - & Cribriform plate \\
\hline 17 & 55 & $\mathrm{~F}$ & Trauma & Operated and cured & - & midline sphenoid \\
\hline 18 & 57 & $\mathrm{~F}$ & Spontaneous & Follow up & - & Lateral sphenoid \\
\hline 19 & 41 & $\mathrm{~F}$ & Spontaneous & Operated and cured & - & Midline sphenoid \\
\hline 20 & 53 & M & Iatrogenic & Operated and cured & Meningoencephalocele & Lateral sphenoid \\
\hline 21 & 43 & M & Trauma & Operated and cured & Meningocele & Midline sphenoid \\
\hline
\end{tabular}

presented with nasal obstruction findings. There were only two meningoencephaloceles in our patients. In one patient, frontal lobe protruded through the ethmoid air cells and in one patient, the right temporal lobe protruded through the sphenoid sinus. The other five cephaloceles were small meningoceles and all presented with watery nasal discharge (Figure 4).

In the literature, several studies reported cribriform plate as the most common location of CSF leakage in spontaneous rhinorrhea patients (20-23). The other possible 

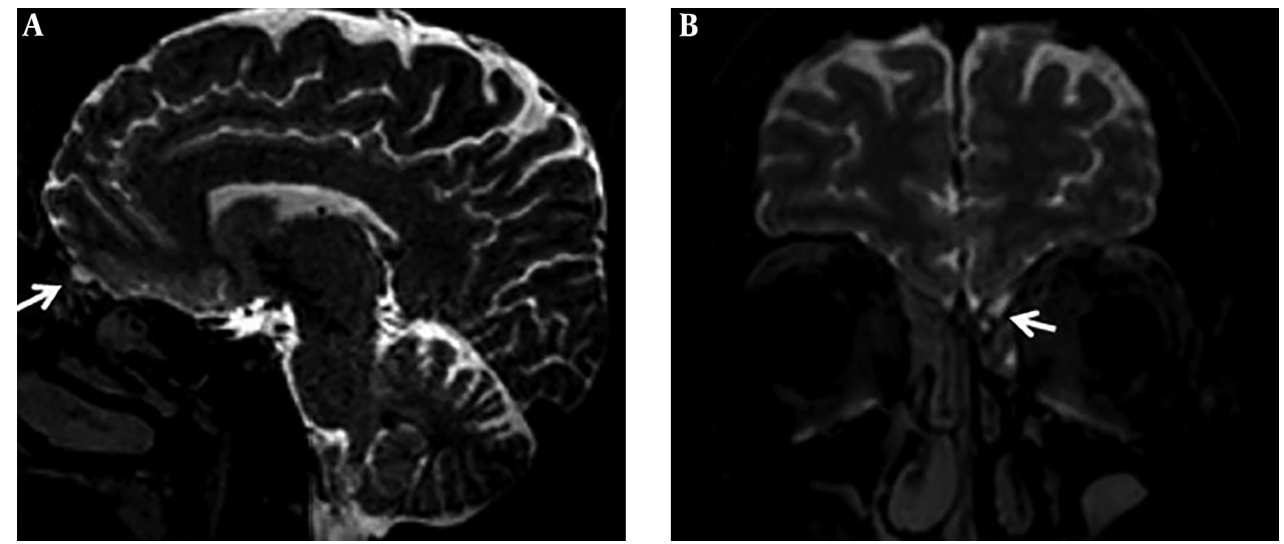

Figure 4. Intrathecal gadolinium enhanced MR cisternography examination in a 47-year-old male patient with persistent watery nasal discharge. Sagittal (A) and coronal (B) three dimensional fluid attenuated inversion recovery (FLAIR) images reveal a small meningocele (arrows).

sites for spontaneous CSF fistulas were craniopharyngeal canal, sella and sphenooccipital synchondrosis. Arachnoid granulations near the ethmoid and sphenoid sinus were seen responsible for meningocele development(23). In our series, three of five spontaneous CSF leakages were in the cribriform plate, one midline sphenoid and one was in the lateral sphenoid location. Selcuk et al. reported ethmoid sinuses as the common location of CSF leakage in their study with Gd-MR cisternography in rhinorrhea patients. Locations of CSF leakage in our series including 21 rhinorrhea patients were compatible with the literature (9).

This study had several limitations. Its retrospective design and limited number of patient groups was the first limitation. Second, five of 21 patients did not undergo endoscopic diagnosis. Third, MRI protocol in this study did not include precontrast images. To our knowledge, there is only one report evaluating Gd-MR cisternography with gadoterate meglumine, and this is the first study evaluating CSF leaks with Gd-MR cisternography using intrathecal gadoterate meglumine (17).

We conclude that MR cisternography with intrathecal administration of gadoterate meglumine is an effective, minimal invasive and safe diagnostic method inducing admissible adverse effects for evaluating CSF leakage in patients with suspected rhinorrhea. Multiplanar imaging capability of Gd-MR cisternography without ionizing radiation exposure provides high sensitivity and specificity in detecting CSF leakage. Cephaloceles must be kept in mind as a cause in non-traumatic rhinorrhea. As a supplement to CT imaging, Gd-MR cisternography enables detection of CSF leakage location and also meningeal and cerebral parenchyma protrusion.

\section{Footnotes}

Authors' Contributions: Study concept and design: Bahattin Hakyemez, Hakan Cebeci, and Selcuk Yilmazlar; analysis and interpretation of data: Bahattin Hakyemez, Selman Candan, Cem Bilgin and Hakan Cebeci; drafting of the manuscript: Hakan Cebeci; critical revision of the manuscript for important intellectual content: Hakan Cebeci, and Bahattin Hakyemez; statistical analysis: Hakan Cebeci

Conflict of Interests: Authors have no financial interests related to the material in the manuscript.

Ethical Approval: The study was confirmed by the Ethical Committee of Uludag University Medical Faculty. The ethical approval code is 2018-16/14.

Funding/Support: This study had not received any funding.

\section{References}

1. Pianta L, Pinelli L, Nicolai P, Maroldi R. Cerebrospinal fluid leak, meningocele and meningoencephalocele. In: Maroldi R, Nicolai P, editors. Imaging in treatment planning for sinonasal diseases. Medical radiology (diagnostic imaging). Berlin, Heidelberg: Springer; 2005. p. 93106. doi: 10.1007/3-540-26631-3_7.

2. Manelfe C, Cellerier P, Sobel D, Prevost C, Bonafe A. Cerebrospinal fluid rhinorrhea: Evaluation with metrizamide cisternography. $A J R$ Am J Roentgenol. 1982;138(3):471-6. doi: 10.2214/ajr.138.3.471. [PubMed: 6977995].

3. Hayashi Y, Iwato M, Kita D, Fukui I. Spontaneous cerebrospinal fluid leakage through fistulas at the clivus repaired with endoscopic endonasal approach. Surg Neurol Int. 2015;6:106. doi: 10.4103/21527806.158898. [PubMed: 26110086]. [PubMed Central: PMC4476136].

4. Goel G, Ravishankar S, Jayakumar PN, Vasudev MK, Shivshankar JJ, Rose D, et al. Intrathecal gadolinium-enhanced magnetic resonance cisternography in cerebrospinal fluid rhinorrhea: Road ahead? J Neurotrauma. 2007;24(10):1570-5. doi: 10.1089/neu.2007.0326. [PubMed: 17970620]. 
5. Aydin K, Guven K, Sencer S, Jinkins JR, Minareci O. MRI cisternography with gadolinium-containing contrast medium: Its role, advantages and limitations in the investigation of rhinorrhoea. Neuroradiology. 2004;46(1):75-80. doi: 10.1007/s00234-003-1004-2. [PubMed: 14615855].

6. Sand T, Myhr G, Stovner LJ, Dale LG. Side effects after lumbar iohexol myelography. Relation to radiological diagnosis, sex and age. Neuroradiology. 1990;31(6):523-8. doi: 10.1007/bf00340134. [PubMed 2352635].

7. Algin O, Hakyemez B, Gokalp G, Ozcan T, Korfali E, Parlak M. The contribution of 3D-CISS and contrast-enhanced MR cisternography in detecting cerebrospinal fluid leak in patients with rhinorrhoea. Br J Radiol. 2010;83(987):225-32. doi: 10.1259/bjr/56838652. [PubMed: 19723768]. [PubMed Central: PMC3473545].

8. Aydin K, Terzibasioglu E, Sencer S, Sencer A, Suoglu Y, Karasu A, et al. Localization of cerebrospinal fluid leaks by gadoliniumenhanced magnetic resonance cisternography: A 5-year single-center experience. Neurosurgery. 2008;62(3):584-9. discussion 584-9. doi 10.1227/01.neu.0000317306.39203.24. [PubMed:18425007].

9. Selcuk H, Albayram S, Ozer H, Ulus S, Sanus GZ, Kaynar MY, et al Intrathecal gadolinium-enhanced MR cisternography in the evaluation of CSF leakage. AJNR Am J Neuroradiol. 2010;31(1):71-5. doi 10.3174/ajnr.A1788. [PubMed: 19762462].

10. La Fata V, McLean N, Wise SK, DelGaudio JM, Hudgins PA. CSF leaks: correlation of high-resolution $\mathrm{CT}$ and multiplanar reformations with intraoperative endoscopic findings. AJNR Am J Neuroradiol. 2008;29(3):536-41. doi: 10.3174/ajnr.A0885. [PubMed: 18079188].

11. Hegarty SE, Millar JS. MRI in the localization of CSF fistulae: Is it of any value? Clin Radiol. 1997;52(10):768-70. doi: 10.1016/s00099260(97)80157-2. [PubMed: 9366537].

12. Di Chiro G, Knop RH, Girton ME, Dwyer AJ, Doppman JL, Patronas NJ, et al. MR cisternography and myelography with Gd-DTPA in monkeys. Radiology.1985;157(2):373-7.doi:10.1148/radiology.157.2.4048444. [PubMed: 4048444]

13. Jinkins JR, Williams RF, Xiong L. Evaluation of gadopentetate dimeglumine magnetic resonance cisternography in an animal model: Preliminary report. Invest Radiol. 1999;34(2):156-9. doi: 10.1097/00004424-199902000-00009. [PubMed: 9951796].

14. Ecin G, Oner AY, Tokgoz N, Ucar M, Aykol S, Tali T. T2-weighted vs. intrathecal contrast-enhanced MR cisternography in the evaluation of CSF rhinorrhea. Acta Radiol. 2013;54(6):698-701. doi: 10.1177/0284185113478008. [PubMed: 23612427].

15. Ragheb AS, Mohammed FF, El-Anwar MW. Cerebrospinal fluid rhinorrhea: Diagnostic role of gadolinium enhanced MR cisternography. Egypt J Radiol Nucl Med. 2014;45(3):841-7. doi: 10.1016/j.ejrnm.2014.05.010

16. Hantula $\mathrm{O}$. The safety and clinical applications of intrathecal gadoliniumenhanced MRI: A systematic review and case report [dissertation]. Tampere University; 2017.

17. Ozturk K, Nas OF, Soylu E, Hakyemez B. Signal changes in the dentate nucleus and globus pallidus on unenhanced T1-weighted magnetic resonance images after intrathecal administration of macrocyclic gadolinium contrast agent. Invest Radiol.2018;53(9):535-40. doi: 10.1097/RLI.0000000000000472. [PubMed: 29727401].

18. Beckhardt RN, Setzen M, Carras R. Primary spontaneous cerebrospinal fluid rhinorrhea. Otolaryngol Head Neck Surg. 1991;104(4):425-32. doi: 10.1177/019459989110400402. [PubMed: 1903854].

19. Mahapatra AK, Suri A. Anterior encephaloceles: A study of 92 cases. Pediatr Neurosurg. 2002;36(3):113-8. doi: 10.1159/000048365. [PubMed: 11919444].

20. Stone JA, Castillo M, Neelon B, Mukherji SK. Evaluation of CSF leaks: high-resolution CT compared with contrast-enhanced CT and radionuclide cisternography. AJNR Am J Neuroradiol. 1999;20(4):706-12. [PubMed: 10319986].

21. Shetty PG, Shroff MM, Sahani DV, Kirtane MV. Evaluation of highresolution CT and MR cisternography in the diagnosis of cerebrospinal fluid fistula. AJNR Am J Neuroradiol. 1998;19(4):633-9. [PubMed: 9576647].

22. Sillers MJ, Morgan CE, el Gammal T. Magnetic resonance cisternography and thin coronal computerized tomography in the evaluation of cerebrospinal fluid rhinorrhea. Am J Rhinol. 1997;11(5):387-92. doi: 10.2500/105065897781286052. [PubMed: 9768321].

23. Schuknecht B, Simmen D, Briner HR, Holzmann D. Nontraumatic skull base defects with spontaneous CSF rhinorrhea and arachnoid herniation: Imaging findings and correlation with endoscopic sinus surgery in 27 patients. AJNR Am J Neuroradiol. 2008;29(3):542-9. doi: 10.3174/ajnr.A0840. [PubMed: 18079185]. 